\section{Interfering with lupus}

\section{By Tim Fulmer, Senior Writer}

This year, the lupus space has seen a pair of high-profile setbacks, as both Rituxan rituximab and Riquent abetimus failed in Phase III trials. The former worked by depleting B cells, whereas the latter targeted autoantibodies. A team of French and American researchers now have an interferon-based lupus vaccine that has shown efficacy in animals ${ }^{1}$ and could provide an alternative way to attack the disease.

Neovacs S.A. has already patented the vaccine and hopes to begin Phase I/II testing by year end. The unanswered question is how the vaccine will stack up in comparison to an anti-interferon antibody that is already in the clinic for lupus.

Lupus is a chronic autoimmune disease characterized by excess production of autoantibodies and proinflammatory cytokines that cause severe damage to the skin, kidneys, nervous system, blood and other organs. ${ }^{2}$ The variety of immune cells involved means there are multiple potential therapeutic targets, although there are no targeted therapies marketed to treat the disease (see Figure 1, “Targeting interferon- $\alpha$ in lupus"). ${ }^{3}$

Previous work has shown that high levels of the proinflammatory cytokine interferon- $\alpha$ (IFN- $\alpha$ ) can play an important role in lupus pathogenesis. ${ }^{4-6}$ Thus, researchers at several French institutes and universities and the University of Maryland, Baltimore hypothesized that vaccination with an IFN- $\alpha$-derived antigen could generate anti-IFN- $\alpha$ antibodies that neutralize systemic IFN- $\alpha$ and thus help treat disease.

In a paper in the Proceedings of the National Academy of Sciences, the team reported that vaccination with a murine IFN- $\alpha$-derived synthetic immunogen-termed an IFN- $\alpha$ kinoid-induced high levels of anti-IFN- $\alpha$ antibodies in a mouse model of lupus. Following IFN- $\alpha$ challenge to simulate a lupus flare, vaccinated mice had delayed onset of proteinuria - a hallmark of lupus-and $50 \%$ survival compared with littermates that received control antigen or saline, with the comparators showing $10 \%$ or $0 \%$ survival, respectively. Postmortem tissue analysis revealed less lupus-related kidney pathology in the vaccinated mice than that seen in the controls.

\section{Balancing safety and efficacy}

Researchers polled by SciBX agreed that targeting IFN- $\alpha$ has good prospects in lupus. The challenge will be choosing a strategy with a good therapeutic index.

Neovacs CEO Guy-Charles Fanneau de La Horie told SciBX that a human version of the IFN- $\alpha$ kinoid vaccine is now in safety and immunogenicity testing in nonhuman primates. A key issue is likely to be determining the proper level of IFN- $\alpha$ blockade to decrease lupus symptoms without increasing susceptibility to opportunistic viral infection.

"Any strategy that seeks to block interferon- $\alpha$ should have its impact on immunity closely monitored-in particular, increased patient susceptibility to viral infections," said Jill Buyon, professor of medicine and associate director of the Division of Rheumatology at the New York University School of Medicine.

Nevertheless, Buyon said the strategy is a good one, as IFN- $\alpha$ is a cytokine that "sits at the juncture of plasmacytoid dendritic cells, differentiation of monocytes and activation of autoreactive T and B cells. Blocking the action of interferon- $\alpha$ in this autoimmune context could indeed have a disease-modifying effect."

"The PNAS paper adds further support to the growing realization that interferon- $\alpha$ is a key player in the lupus disease process. The question is how to block the cytokine safely," said Sudhir Agrawal, CEO and CSO of Idera Pharmaceuticals Inc. "A preventative vaccine could shut down nearly all interferon- $\alpha$, whereas an antibody would likely have a more transient effect on interferon- $\alpha$ levels. The latter approach might thus be preferable to the former only because it could have a wider safety margin."

In the PNAS paper, the authors did not report cases of opportunistic infections. They concluded that the vaccine was safe because the anti-IFN$\alpha$ antibodies it induced did not interfere with cytokine function in healthy tissues and elicited transient IFN- $\alpha$ inhibition.

Another concern is how well the vaccine works in a combination therapy setting, said Barbara White, VP of clinical development at AstraZeneca plc's MedImmune Inc. subsidiary. "Lupus patients are generally on long-term immunosuppressants. The dampening effect of those drugs on the patient's immune system could potentially have an impact on how well the patients respond to the vaccine," she said.

MEDI-545, a mAb targeting IFN- $\alpha$ from partners Medarex Inc. and MedImmune, is in Phase II testing to treat systemic lupus erythematosus (SLE).

According to White, any differences between the antibody and vaccine approaches will likely relate to safety rather than efficacy. "So long as each approach generates a sufficient level of anti-interferon- $\alpha$ antibody, they could show comparable efficacy against the disease. One potential advantage of the recombinant antibodies is that it may be easier to deliver them at a fixed dose and maintain proper serum levels over time compared with relying on a vaccination and booster regimen," she said.

Indeed, the authors of the PNAS paper noted that vaccinated mice did not mount identical immune responses against IFN- $\alpha$. Instead, animals fell into one of two groups: responders and low or nonresponders. Moreover, the anti-IFN- $\alpha$ antibody response was generally transient and declined rapidly. Thus, the authors speculated that "to maintain a long-term Ab-dependent clinical benefit triggered by kinoid immunization, periodic booster injections (2-4 per year) will likely be required."

Idera's approach is to target toll-like receptors (TLRs) in lupus.

"Two cell types are implicated to play a role in lupus-plasmacytoid dendritic cells that secrete interferon- $\alpha$ and B cells that secrete 


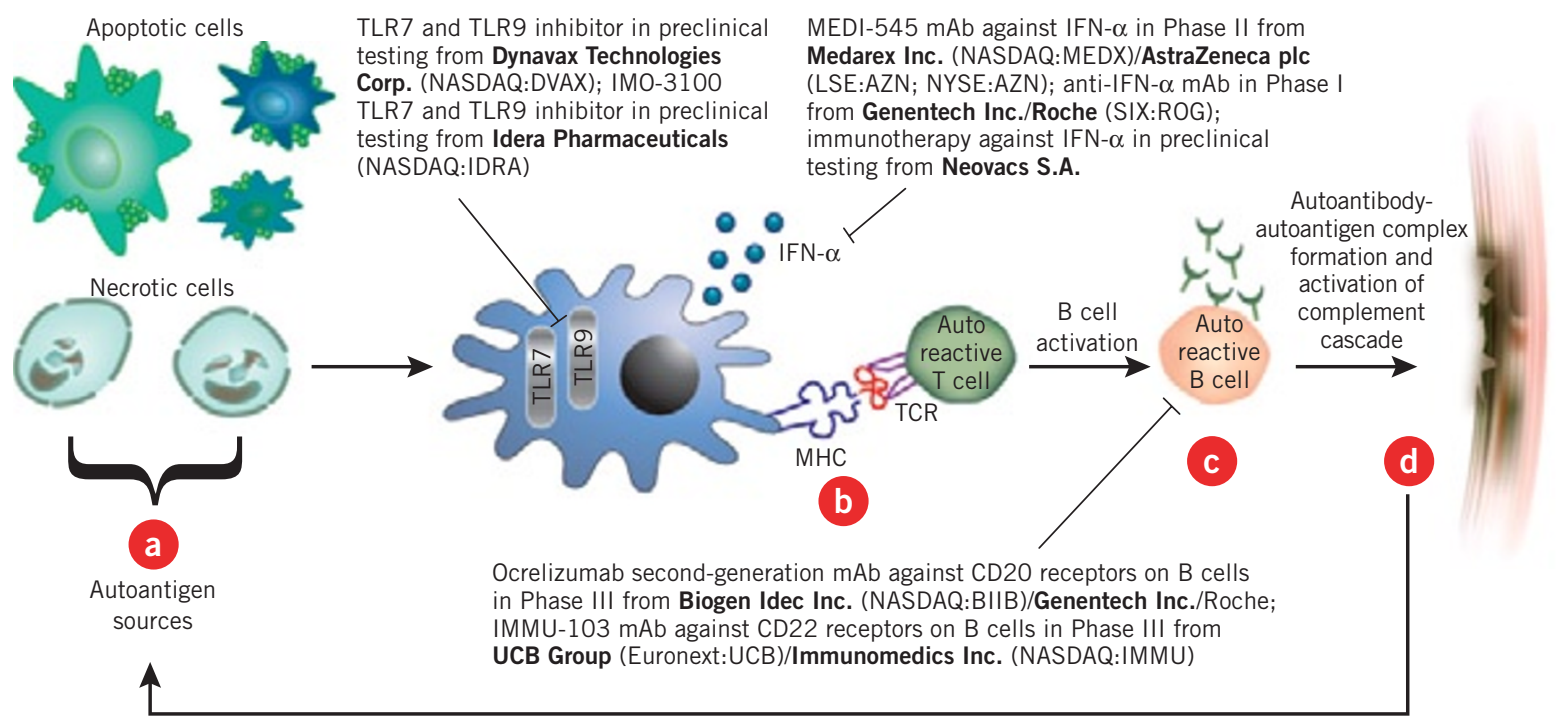

Figure 1. Targeting interferon- $\alpha$ in lupus. A paper published in the Proceedings of the National Academy of Sciences suggests that inducing the production of host antibodies against interferon- $\alpha$ (IFN- $\alpha$ ) by vaccination could help treat lupus, a disease characterized by high levels of autoantibodies that potentially cause damage to multiple tissues.

[a] In lupus patients, debris from apoptotic and necrotic cells can provide a source of autoantigens such as double-stranded DNA.

[b] Those autoantigens then bind to toll-like receptors (TLRs) on antigen-presenting cells such as plasmacytoid dendritic cells (pDCs). This leads to increased production of IFN- $\alpha$ as well as activation of autoreactive T cells when T cell receptors (TCRs) bind the MHC complex. IFN- $\alpha$ can then potentially stimulate multiple immune cells involved in the antiviral and autoimmune response.

[c] The autoreactive T cells activate autoreactive B cells, which secrete autoantibodies.

[d] In multiple tissues and organs, the autoantibodies bind autoantigens, forming immune complexes, activating the complement cascade and ultimately causing tissue damage and organ failure.

By inducing the production of antibodies against IFN- $\alpha$, the lupus vaccine thus potentially prevents stimulation of multiple immune cells that can contribute to autoimmune diseases like lupus.

antibodies," said Agrawal. "Both of those cell types express TLR7 and TLR9, which mediate the secretion of proinflammatory factors in response to complexes of autoantigens and nucleic acids. By blocking TLRs at the cellular level with antagonists, our strategy shuts down production of autoantibodies and cytokines like interferon- $\alpha$ without impairing the host antiviral response."

Idera plans to file an IND in 2H09 for its lead TLR7 and TLR9 antagonist, IMO-3100, to treat autoimmune diseases, including lupus.

New strategies are wanted in the wake of this year's late-stage setbacks.

A Phase III lupus nephritis trial of Riquent abetimus (LJP 394), from La Jolla Pharmaceutical Co. and BioMarin Pharmaceutical Inc., was halted earlier this year based on an interim efficacy analysis. The treatment combined a carrier plus four double-stranded oligonucleotides that bind to anti-double-stranded DNA autoantibodies.

Last month, Rituxan rituximab (R105), a chimeric mAb against CD20 antigen-expressing B cells from Biogen Idec Inc., missed the primary endpoint of decreasing disease at 52 weeks in a Phase III lupus nephritis trial.

Rituxan is marketed for non-Hodgkin's lymphoma and rheumatoid arthritis (RA) by Biogen Idec and the Genentech Inc. unit of Roche in the U.S., whereas Roche markets it elsewhere as MabThera.
Fulmer, T. SciBX 2(13); doi:10.1038/scibx.2009.519

Published online April 2, 2009

\section{REFERENCES}

1. Zagury, D. et al. Proc. Natl. Acad. Sci. USA; published online March 2, 2009; doi:10.1073/pnas.0900615106

Contact: Daniel Zagury, Neovacs S.A., Paris, France e-mail: dzagury@neovacs.com

Contact: Robert Gallo, University of Maryland, Baltimore, Md. e-mail: rgallo@ihv.umaryland.edu

2. Mok, C.C. \& Lau, C.S. J. Clin. Pathol. 56, 481-490 (2003)

3. Mount, G. \& Gilliland, W. Clin. Pharmacol. Ther. 83, 167-171 (2008)

4. Mathian, A. et al. J. Immunol. 174, 2499-2506 (2005)

5. Baechler, E. et al. Proc. Natl. Acad. Sci. USA 100, 2610-2615 (2003)

6. Bennett, L. et al. J. Exp. Med. 17, 711-723 (2003)

\section{COMPANIES AND INSTITUTIONS MENTIONED}

AstraZeneca plc (LSE:AZN; NYSE:AZN), London, U.K.

Biogen Idec Inc. (NASDAQ:BIIB), Cambridge, Mass.

BioMarin Pharmaceutical Inc. (NASDAQ:BMRN), Novato, Calif. Genentech Inc. (NYSE:DNA), South San Francisco, Calif.

Idera Pharmaceuticals Inc. (NASDAQ:IDRA), Cambridge, Mass. La Jolla Pharmaceutical Co. (NASDAQ:LJPC), San Diego, Calif.

Medarex Inc. (NASDAQ:MEDX), Princeton, N.J.

Medlmmune Inc., Gaithersburg, Md.

Neovacs S.A., Paris, France

New York University School of Medicine, New York, N.Y.

Roche (SIX:ROG), Basel, Switzerland

University of Maryland, Baltimore, Md. 